\title{
Antiangiogenic agent, thalidomide increases the antitumor effect of single high dose irradiation (gamma knife radiosurgery) in the rat orthotopic glioma model
}

\author{
JUNG-IL LEE $^{1 *}$, SATOSHI ITASAKA ${ }^{3 *}$, JI TAE KIM $^{1,2}$ and DO-HYUN NAM ${ }^{1}$ \\ ${ }^{1}$ Department of Neurosurgery, Samsung Medical Center and Samsung Biomedical Research Institute, \\ Sungkyunkwan University School of Medicine; ${ }^{2}$ Laboratory of Radiation Cytogenetics and Epidemiology, \\ Korea Institute of Radiological and Medical Sciences, Seoul, Korea; ${ }^{3}$ Radiation Oncology \\ and Image-Applied Therapy, Graduate School of Medicine, Kyoto University, Kyoto, Japan
}

Received November 30, 2005; Accepted January 18, 2006

\begin{abstract}
Gliomas are primary brain tumors associated with a poor prognosis partly due to resistance to conventional therapies. To overcome this problem, we investigated the combined effects of gamma knife radiosurgery (GKS) and an antiangiogenic agent, thalidomide (THD), or a chemotherapeutic agent, temozolomide (TMZ), on a rat glioma model. GKS (20 Gy single dose) alone and/or drugs (for 3 days) were delivered 14 or 18 days after stereotactic implantation of C6/LacZ glioma cells into the brains of Sprague-Dawley rats. A group of animals treated with or without drugs for 3 days was irradiated on day 18 and sacrificed at $24 \mathrm{~h}$ after GKS to evaluate cell proliferation, apoptosis and microvessel density. The other group of animals was irradiated on day 14 and sacrificed at day 5 after GKS for the measurement of tumor volume. Apoptosis of endothelial cells in the tumor beds was only observed in the early period after GKS. Decreased cell proliferation and increased tumor cell apoptosis were observed in rat gliomas treated with GKS and THD or TMZ. The combination treatments with GKS and THD or GKS and TMZ also decreased microvessel density, i.e. angiogenesis, more effectively compared with GKS treatment alone. The combination of GKS and THD was the most effective regimen, resulting in a significant decrease of tumor volume. We suggest that the antitumor effect of GKS on glioma is enhanced by the addition of THD. Therefore, combined therapy with GKS and THD might be a favorable treatment for gliomas.
\end{abstract}

Correspondence to: Dr Do-Hyun Nam, Department of Neurosurgery, Samsung Medical Center and Samsung Biomedical Research Institute, Sungkyunkwan University School of Medicine, 50 Irwon-dong, Gangnam-gu, Seoul 135-710, Korea

E-mail: nsnam@smc.samsung.co.kr

${ }^{*}$ Contributed equally

Key words: gamma knife radiosurgery, glioma, thalidomide, antiangiogenic treatment, orthotopic model

\section{Introduction}

Malignant gliomas are the most common primary brain tumors associated with a high degree of morbidity and mortality (1). Chemoradiation therapy remains a popular adjuvant modality after gross tumor resection. Although the combination treatment of radiotherapy and chemotherapy has improved survival $(2,3)$, the recurrence rate is still high and prognosis is poor.

Stereotactic radiosurgery (gamma knife radiosurgery, GKS) is a radiation technique characterized by the accurate delivery of high dose irradiation to a small volume in a single session. Irradiation dose falls off sharply outside the target. Therefore, radiosurgery can safely be added to the conventional radiation therapy to improve local control. However, the addition of radiosurgery to conventional radiotherapy with carmustine did not improve outcome in a randomized clinical trial (4). Thus, new therapeutic strategies other than the simple addition of radiosurgery are necessary to improve the survival of patients with malignant gliomas.

Vascular targeted therapy, i.e. antiangiogenic therapy that can inhibit endothelial proliferation or induce endothelial cell apoptosis in tumor beds, has been the focus of many studies. It was also reported that tumor endothelial cells play an important role in the tumor response to irradiation $(5,6)$.

Gliomas are the most angiogenic human tumors, as characterized by proliferative vascular endothelial cells with high levels of cytokines such as basic fibroblastic growth factor (bFGF) and vascular endothelial cell growth factor (VEGF) (7-10). The high expression levels of VEGF and bFGF contribute to the enhanced survival of tumor-associated endothelial cells, and results in a certain level of tumor resistance to irradiation (11).

Thalidomide (THD), an antiangiogenic agent, is effective for the treatment of multiple myeloma and is being tested on various types of cancers (12). It is thought to have a therapeutic effect by inhibiting VEGF- $(13,14)$ and bFGF- $(15)$ induced angiogenesis. There are several clinical reports suggesting the efficacy of THD for treating recurrent malignant glioma $(16,17)$. In addition, it has been suggested that the combination 
Table I. Determination of ideal radiosurgery dose.

TUNEL-positive cells

\begin{tabular}{ccccc} 
Maximum dose & $90 \%$ isodose & Microvessel density (MVD) & Periventricle & Basal ganglia \\
\hline $160 \mathrm{~Gy}$ & $144.0 \mathrm{~Gy}$ & $28.3 \pm 5.9^{\mathrm{a}}$ & $46.7 \pm 13.3^{\mathrm{b}}$ & $10.8 \pm 3.8^{\mathrm{a}}$ \\
$80 \mathrm{~Gy}$ & $72.0 \mathrm{~Gy}$ & $31.3 \pm 9.0^{\mathrm{b}}$ & $33.8 \pm 24.8^{\mathrm{b}}$ & $5.8 \pm 1.2^{\mathrm{b}}$ \\
$40 \mathrm{~Gy}$ & $36.0 \mathrm{~Gy}$ & $45.2 \pm 12.2^{\mathrm{b}}$ & $22.5 \pm 7.3$ & $3.8 \pm 1.2$ \\
$20 \mathrm{~Gy}$ & $18.0 \mathrm{~Gy}$ & $53.8 \pm 10.2$ & $12.5 \pm 6.3$ & $3.5 \pm 1.2$ \\
$10 \mathrm{~Gy}$ & $9.0 \mathrm{~Gy}$ & $63.2 \pm 7.2$ & $9.7 \pm 11.7$ & $3.8 \pm 1.2$ \\
$5 \mathrm{~Gy}$ & $4.5 \mathrm{~Gy}$ & $59.3 \pm 5.6$ & $3.7 \pm 4.7$ & $3.3 \pm 0.4$ \\
$0 \mathrm{~Gy}$ & $0.0 \mathrm{~Gy}$ & $3.0 \pm 0.7$ & $1.3 \pm 0.9$ \\
\hline
\end{tabular}

After normal rat brains received the indicated doses of GKS, microvessel density (MVD) and apoptosis were evaluated by immunostaining for CD31 and TUNEL. Each value is the number of respective stained cells, representing the mean \pm SD from five animals for each group. Lower (MVD) or higher (TUNEL) than the control (rats receiving 0 Gy of GKS), ${ }^{a} \mathrm{P}<0.001$ and ${ }^{\mathrm{b}} \mathrm{P}<0.05$.

regimens with antiangiogenic therapy and radiotherapy increase antitumor effect $(7,18,19)$.

Temozolomide (TMZ) is an alkylating agent that exhibits broad-spectrum antitumor activity (20). Currently, TMZ is the most widely used agent for progressive or recurrent malignant glioma, and concomitant TMZ treatment during radiotherapy for newly diagnosed glioblastoma was associated with a better survival $(21,22)$.

In this study, we combined the antiangiogenic THD or chemotherapeutic TMZ agent with the single high dose of GKS radiation to evaluate the possible synergistic effect of radiosurgery and the above agents. If vascular endothelial cell apoptosis is a major event preceding tumor cell apoptosis after irradiation, the combination of GKS with antiangiogenic therapy may more effectively induce tumor shrinkage than that with chemotherapy. We investigated the antiangiogenic and antitumor effects of combined treatment with GKS and THD (GKS+THD) in comparison with those of GKS and TMZ (GKS+TMZ).

\section{Materials and methods}

Animals and orthotopic implantation of tumor cells. Specific pathogen-free Sprague-Dawley (SD) rats were used. All animal work was approved by the Animal Care and Use Committee of the Samsung Medical Center. Orthotopic implantation of glioma cells was performed as described previously (23). Briefly, male SD rats (200-250 g) were anesthetized and shaved. They were secured in a rodent stereotactic frame, and a hollow guide screw was implanted into a small drill hole made $3 \mathrm{~mm}$ left lateral and $1 \mathrm{~mm}$ anterior to the bregma. (24). C6/LacZ, rat glioma cells $\left(1 \times 10^{5}\right)$ grown in Dulbecco's modified essential medium supplemented with $10 \%$ fetal bovine serum and antibiotics were injected through the hollow guide screw into the white matter at a depth of $5 \mathrm{~mm}$.

Gamma knife radiosurgery ( $G K S$ ). Radiosurgery was performed using the gamma knife model $\mathrm{C}$ (Elekta $\mathrm{AB}$ ) on the 14th or 18th day after tumor cell implantation. The GammaPlan (version 5.34; Elekta AB) was used for dose calculations. A spherical skull radius of $15 \mathrm{~mm}$ was used, and a single shot of radiation was given through an $8 \mathrm{~mm}$ collimator with a maximum dose at the center of tumors by a rate of $310 \mathrm{cGy} / \mathrm{min}$. Tumors were covered with a $90 \%$ isodose line. In detail, the rats were anesthetized and placed in a stereotactic head frame with a position identical to site of tumor cell inoculation. The coordinate for tumor cell inoculation was used as an isocenter coordinate for gamma irradiation. Animals in the control group and those treated with only pharmacologic therapy underwent the same procedure of anesthesia and positioning on the stereotactic frame for GKS without actual irradiation.

Harvesting of specimens. The harvested brains of rats were axially sectioned. One section was fixed in $10 \%$ buffered formalin and embedded in paraffin, and the other was embedded in OCT compound, frozen rapidly in liquid nitrogen, and stored at $-70^{\circ} \mathrm{C}$.

Histological evaluation. Immunohistochemistry was performed as described previously (23). For PCNA, TUNEL, CD31 and nucleus staining, tissue sections were stained with each antibody for PCNA (Dako), a TUNEL kit (Intergen Company), CD31/PECAM-1 (BD Pharmingen) and DAPI (Molecular Probe), respectively. To quantify each immunostaining, the number of stained cells was counted in 10 random fields. The tumor volume was calculated by measuring the section with the largest tumor portion and applying the formula: Length $\mathrm{x}$ width $^{2} \mathrm{x} 0.5$.

Combined therapies of GKS and pharmacotherapeutic agent. TMZ was provided by the Yuhan Corporation, and THD was purchased from Sigma. To elucidate an acute response to combination treatment with GKS and an antiangiogenic or a chemotherapeutic agent on a glioma model, THD (4 mg/ body/day) or TMZ $(7.5 \mathrm{mg} / \mathrm{kg} /$ day $)$ was administered from the 16 th to 18 th day after intracranial implantation. Rats then received irradiation on the 18th day in each treatment group, and the brains of rats were harvested $24 \mathrm{~h}$ after GKS to compare cell proliferation, apoptosis and microvessel density (MVD). To check the antitumor effect of combined treatment, the same amount of drugs were administered orally from the 12 th to 14th day in the other groups, and GKS was done on the 14th day. The brains were harvested 5 days after GKS to compare tumor volume. 
A

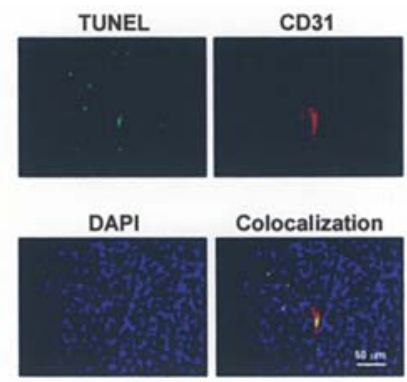

C

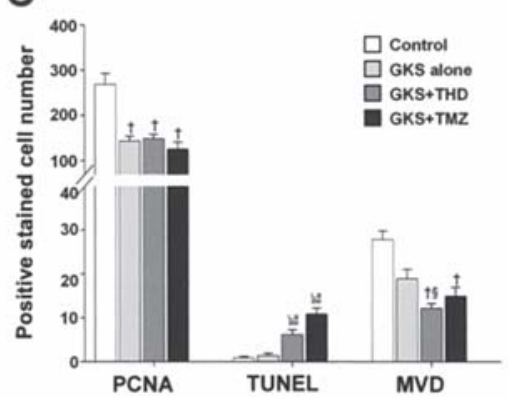

B

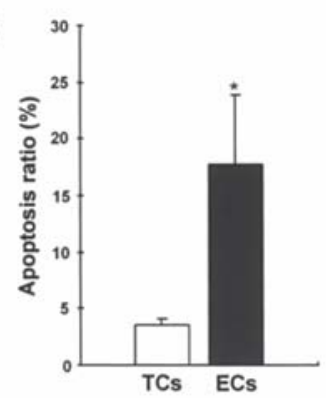

D

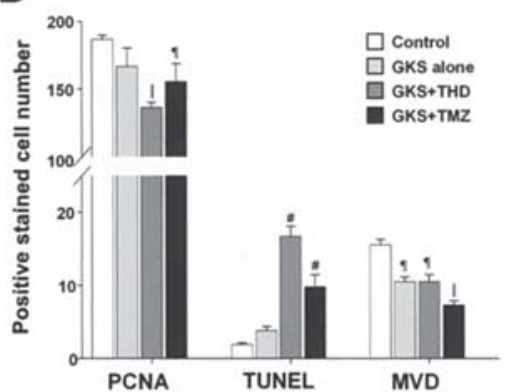

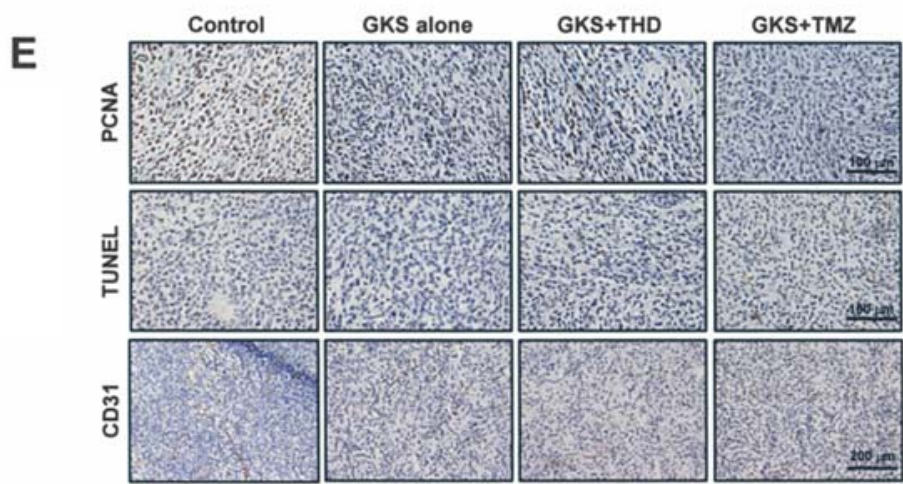

F

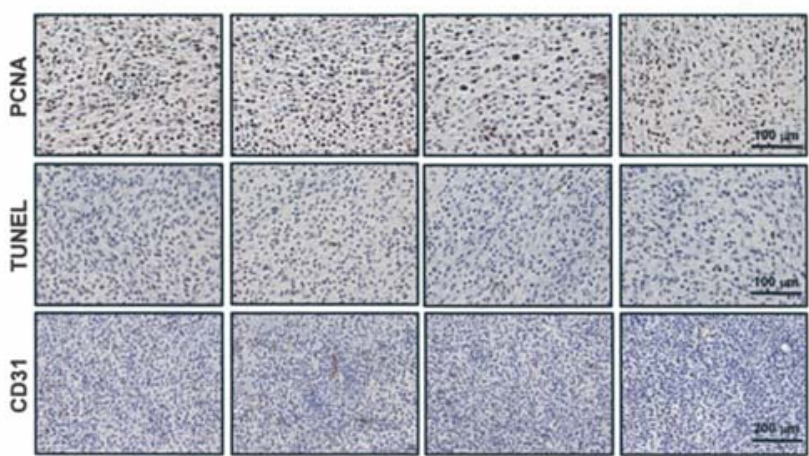

Figure 1. The acute effects of gamma knife radiosurgery (GKS) and/or pharmacotherapy (TMZ or THD) in a C6/LacZ orthotopic rat glioma model. (A) Colocalization of apoptosis (TUNEL, green), endothelial cells (CD31, red) and the nucleus (DAPI, blue) was performed with each antibody staining at $6 \mathrm{~h}$ after GKS on confocal microscopy. Apoptotic endothelial cells are seen as yellow spots in merged images. (B) The apoptosis ratio was calculated by dividing the number of apoptotic cells by that of total cells for each cell type in tumor cells (TCs) and endothelial cells (ECs) of a glioma model at $6 \mathrm{~h}$ after GKS. Each value represents the mean $\pm \mathrm{SD}$. *Higher than in tumor cells $(\mathrm{P}<0.05)$. (C and $\mathrm{D})$ The cell proliferation, apoptosis and microvessel density $(\mathrm{MVD})$ in rat brains implanted with C6/LacZ glioma cells were evaluated by immunostaining for PCNA, TUNEL and CD31 in tumor regions with (C) or without (D) necrosis, respectively. Each value is the number of respective stained cells, representing the mean \pm SD ( $=5-7)$. ${ }^{\dagger}$ Lower $(\mathrm{PCNA}$ or MVD) or higher $(\mathrm{TUNEL})$ than in control animals $(\mathrm{P}<0.001)$. ${ }^{*}$ Higher (TUNEL) than in control animals $(\mathrm{P}<0.05)$. ${ }^{\S}$ Lower than in animals treated with GKS (P<0.05). "Lower than in control and GKS-treated animals $(\mathrm{P}<0.05)$. 'Lower than in control animals $(\mathrm{P}<0.01)$. "Higher than in animals treated with $\mathrm{GKS}(\mathrm{P}<0.01)$. $(\mathrm{E}$ and $\mathrm{F})$ The representative immunostainings for PCNA, TUNEL and CD31 were shown for tumors with (E) or without necrosis (F), respectively. The cells stained brown represent positive cells for each staining.

Data analysis and statistics. Values are presented as means \pm $\mathrm{SD}$ or $\pm \mathrm{SE}$ from $n$ animals. Statistical comparisons between groups were performed using the Student's t-test or ANOVA. Values of $\mathrm{P}<0.05$ were considered statistically significant.

\section{Results}

Dose determination of radiosurgery. To determine the ideal dose of radiosurgery, we examined various dose effects in 
Table II. The effect of GKS and/or pharmacotherapy (TMZ or THD) on tumor volume in rat brain implanted with C6/LacZ rat glioma cells.

Tumor volume $\left(\mathrm{mm}^{3}\right)$

on 5 th day after GKS

\begin{tabular}{lccc}
\cline { 3 - 3 } Group & $\mathrm{n}$ & Median & \multicolumn{1}{c}{ Range } \\
\hline No GKS & & & \\
Control & 5 & 415.8 & $175.5-508.1$ \\
THD & 6 & 170.4 & $38.8-258.0$ \\
TMZ & 8 & 133.3 & $85.8-674.4$ \\
GKS & & & \\
Control & 5 & 221.3 & $42.0-1118.2$ \\
THD & 5 & $13.5^{\mathrm{a}}$ & $11.0-146.1$ \\
TMZ & 5 & 117.0 & $1.0-157.1$ \\
\hline
\end{tabular}

${ }^{a}$ Smaller than in control animals (no treatment) $(\mathrm{P}<0.05)$.

non-tumor-bearing rat brains. Normal rats $(n=5$ per each group) underwent GKS with each maximum dose at the center of sham inoculation site (Table I). Apoptosis and microvessel density of neural cells were evaluated in these groups of animals by TUNEL and CD31 immunostaining, respectively. Doses of more than 20 Gy caused a significant reduction of MVD and an augmentation of apoptosis of neural cells in a dose-dependent manner (Table I). Therefore, we applied a maximal dose of $20 \mathrm{~Gy}$ at the center of the tumor in the following experiments to prevent normal tissues from radiation injury.

Apoptosis of endothelial cells and tumor cells in the early period after GKS. We checked the apoptosis of endothelial and tumor cells in rat brains implanted with glioma cells at 6 ,
12 or $24 \mathrm{~h}$ after GKS. At $6 \mathrm{~h}$ after GKS, the apoptosis of endothelial cells was prominently increased (Fig. 1A), but not at 12 or $24 \mathrm{~h}$ (data not shown). By dividing the number of apoptotic cells by that of total cells for each cell type, the apoptosis ratio $(18.7 \%)$ of endothelial cells in the early period after GKS was determined to be higher than that (3.6\%) of tumor cells (Fig. 1B). These results demonstrated that the apoptosis of vascular endothelial cells precede that of tumor cells.

Acute effect of combination treatment with GKS and THD or $T M Z$ in rat brains implanted with C6/LacZ glioma cells. We examined the acute responses of treatment with GKS alone, GKS+THD or GKS+TMZ in rats implanted with C6/LacZ glioma cells at $24 \mathrm{~h}$ after GKS and compared them with those of control rats. We distinguished the tumors with necrosis from those without since different patterns of biological effects were estimated according to the presence of necrosis. Cell proliferation, apoptosis and MVD were evaluated by PCNA, TUNEL and CD31 immunostaining in both types of tumors, respectively. In tumors with necrosis (Fig. 1C and E), the number of stained PCNA-positive cells was reduced in rats treated with GKS alone by $53 \%$ and combined therapy by $55 \%$ with GKS+THD and $47 \%$ with GKS+TMZ, relative to the controls $(\mathrm{P}<0.001)$. However, the number of apoptotic cells stained in the TUNEL assay was only increased by combined therapy with GKS+THD (7.4-fold increase) or GKS+TMZ (13.0-fold increase) compared with the controls $(\mathrm{P}<0.001)$. Only in rats that received GKS+THD was the number of CD31-stained vessels significantly decreased by $44 \%$ compared with controls $(\mathrm{P}<0.001)$. In tumors without necrosis (Fig. 1D and F), the number of stained PCNApositive cells was remarkably reduced by $73 \%(\mathrm{P}<0.05)$, and the number of apoptotic cells stained in the TUNEL assay was dramatically increased 8.79 -fold $(\mathrm{P}<0.01)$ in rats treated with GKS+THD compared with the controls. However, MVD was not significantly changed in rats that received GKS+THD.
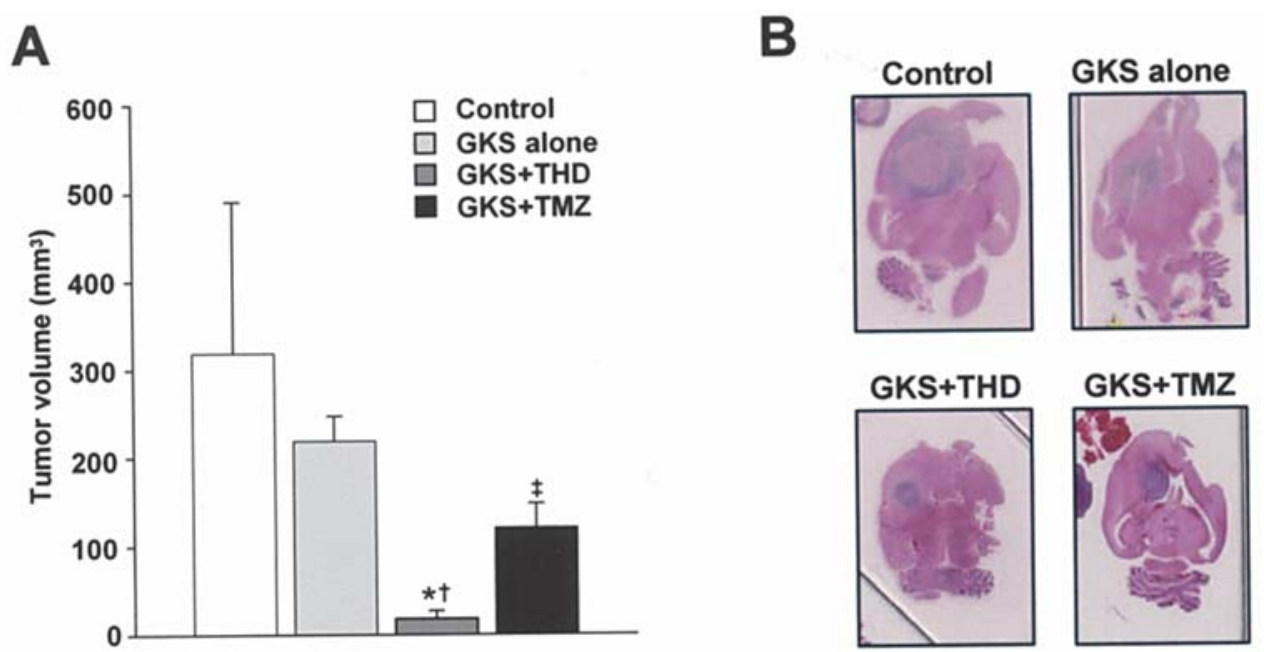

Figure 2. The subacute effects of the control (no treatment), GKS alone, GKS+THD or GKS+TMZ on tumor volume in rat brains implanted with C6/LacZ glioma cells. The tumor volume of each treated group is showm in the graph (A) and displayed with light microscopy (B). Tumor volumes are shown as the mean \pm SE from five rats. The values in this figure (mean) are different from those in Table II (median). ${ }^{*}$ Smaller than in control animals ( $\left.<<0.001\right)$. ${ }^{\dagger}$ Smaller than in animals treated with GKS $(\mathrm{P}<0.05)$. ${ }^{\ddagger}$ Smaller than in control animals $(\mathrm{P}<0.05)$. 
Effect of combination treatment with GKS and THD on tumor volume in rat brains implanted with C6/LacZ glioma cells. To determine the antitumor effects of combination therapy with GKS and TMZ or THD, we monitored tumor size after each treatment in an orthotopic rat glioma model. Five days after GKS, tumor volume was investigated in the groups of animals listed in Table II. The average tumor volume of animals treated with a single regimen of GKS, THD or TMZ were $53 \%, 41 \%$ or $32 \%$ (calculated by median values) smaller than that of controls, respectively (Table II and Fig. 2A). The tumor volume of GKS+THD was $3 \%$ smaller than that of controls. These results demonstrate that the combination treatment of GKS and THD was the most effective in inhibiting tumor growth in this model, suggesting extremely synergistic effects (Table II and Fig. 2B).

\section{Discussion}

Malignant glioma is a tumor accompanied with prominent angiogenesis. Regarding radiation therapy of glioma, it was reported that irradiated C6 glioma cells induce angiogenesis and activation of endothelial cells (11), and glioblastoma cells block radiation-induced apoptosis of endothelial cells (25). These observations provide important implications in developing a novel therapeutic strategy, as a previous report suggests that the sensitivity of tumor vasculature is a major determinant of overall response to radiotherapy (5).

In this study, actual dose at the tumor periphery is estimated at $18 \mathrm{~Gy}$, while the maximum dose at the center of the tumor is $20 \mathrm{~Gy}$. This is equivalent to the dose that causes significant apoptosis of endothelial and tumor cells in other studies $(5,26)$. As predicted, GKS alone resulted in the apoptosis of endothelial cells in the early period after irradiation, which is compatible with the result of another study (27). Increased endothelial cell apoptosis was also observed with the addition of THD. The effective suppression of tumor growth by combined treatment of GKS and THD suggests that the synergistic effects of antiangiogenic agents and irradiation on endothelial cell apoptosis result in improved tumor control. Similar to our data, it was reported that the microenvironmental changes induced by THD were sufficient to radiosensitize tumors in a mouse sarcoma syngeneic model (26).

However, it is not likely that the effects of THD added to irradiation result from a single mechanism. THD has been known to have anticancer effects via antiangiogenesis, proapotosis, the activation of immune response, etc. (12). In the necrotic tumor, there was a significant reduction of the MVD in the GKS+THD group and an increase of tumor cell apoptosis compared with those of the other treatment group. The antiangiogenic effect of THD is thought to be a major cause of synergism, resulting in tumor necrosis and effective tumor volume reduction. Meanwhile, in rapid growing tumors without necrosis, THD reduced the proliferation of tumor cells with an enhanced antitumor effect of GKS, although MVD was not significantly affected. We also confirmed that THD inhibited the expression of VEGF and bFGF in tumors and reduced the ED1-immunopositive cells (28). Decreased expression of these pro-survival cytokines might also enhance the antitumor effect of irradiation through the promotion of tumor cell apoptosis. The synergistic effect of our results may also be explained by reports that the administration of antiangiogenic agent THD might prune the immature blood vessels and consequently result in the normalization of tumor vasculature $(29,30)$.

There are some limitations when these findings are translated to the clinic, i.e. implanted glioma in rats tends to grow with a more circumscribed pattern compared with human glioblastoma, which shows both an expansile and infiltrative growth pattern. Infiltrating tumor cells are difficult to control by surgical excision or stereotactic radiation, which focuses on the macroscopic tumors visible with imaging. However, the promising results of the present study have significant clinical relevance despite some limitations and can be applied to patients. It is traditionally assumed that the biological effect of single dose irradiation is equivalent to fractionated irradiation at a higher total dose (biologically equivalent dose, BED). BED for normal endothelial cells is known to be higher than malignant tumor cells, as demonstrated by the clinical outcome of arteriovenous malformations in which radiosurgery is advantageous over fractionated irradiation. The biological characteristics of endothelial cells incorporated in the tumor may be different from that in a normal environment, particularly when exposed to irradiation. Combination with antiangiogenic agents may restore the responsiveness of endothelial cells to irradiation, whereas single high dose irradiation may be more effective than multiple low doses of irradiation. In conclusion, it is considered that although radiosurgery combined with antiangiogenic therapy needs to be further investigated not only in animal models, but also in clinical trials, it might be an excellent potential therapy for malignant gliomas.

\section{Acknowledgements}

This work was supported by a grant from the Samsung Biomedical Research Institute (C-A5-225-1 to J.-I. Lee and C-A4-208-2 to D.-H. Nam), In-Sung Foundation for Medical Research (CA4835 to J.-I. Lee), and Korea Health 21 R\&D Project, Ministry of Health and Welfare, Republic of Korea (project no. 0405-B002-0205-0001). We thank Electa Korea for its support and Dr Jin Hye Seo for critically reviewing the manuscript.

\section{References}

1. Prados MD and Levin V: Biology and treatment of malignant glioma. Semin Oncol 27: 1-10, 2000.

2. Fine HA, Dear KB, Loeffler JS, Black PM and Canellos GP: Meta-analysis of radiation therapy with and without adjuvant chemotherapy for malignant gliomas in adults. Cancer 71: 2585-2597, 1993.

3. Stewart LA: Chemotherapy in adult high-grade glioma: a systematic review and meta-analysis of individual patient data from 12 randomised trials. Lancet 359: 1011-1018, 2002.

4. Souhami L, Seiferheld W, Brachman D, Podgorsak EB, Werner-Wasik M, Lustig R, Schultz CJ, Sause W, Okunieff P, Buckner J, Zamorano L, Mehta MP and Curran WJ Jr: Randomized comparison of stereotactic radiosurgery followed by conventional radiotherapy with carmustine to conventional radiotherapy with carmustine for patients with glioblastoma multiforme: report of Radiation Therapy Oncology Group 93-05 protocol. Int J Radiat Oncol Biol Phys 60: 853-860, 2004.

5. Garcia-Barros M, Paris F, Cordon-Cardo C, Lyden D, Rafii S, Haimovitz-Friedman A, Fuks Z and Kolesnick R: Tumor response to radiotherapy regulated by endothelial cell apoptosis. Science 300: 1155-1159, 2003. 
6. Moeller BJ, Cao Y, Li CY and Dewhirst MW: Radiation activates HIF-1 to regulate vascular radiosensitivity in tumors: role of reoxygenation, free radicals, and stress granules. Cancer Cell 5: 429-441, 2004.

7. Gorski DH, Beckett MA, Jaskowiak NT, et al: Blockage of the vascular endothelial growth factor stress response increases the antitumor effects of ionizing radiation. Cancer Res 59: 3374-3378, 1999.

8. Geng L, Donnelly E, McMahon G, Lin PC, Sierra-Rivera E, Oshinka $\mathrm{H}$ and Hallahan DE: Inhibition of vascular endothelial growth factor receptor signaling leads to reversal of tumor resistance to radiotherapy. Cancer Res 61: 2413-2419, 2001.

9. Hess C, Vuong V, Hegyi I, Riesterer O, Wood J, Fabbro D Glanzmann C, Bodis S and Pruschy M: Effect of VEGF receptor inhibitor PTK787/ZK222584 (correction of ZK222548) combined with ionizing radiation on endothelial cells and tumour growth. Br J Cancer 85: 2010-2016, 2001

10. Kaur B, Tan C, Brat DJ, Post DE and Van Meir EG: Genetic and hypoxic regulation of angiogenesis in gliomas. J Neurooncol 70: 229-243, 2004.

11. Parthymou A, Kardamakis D, Pavlopoulos I and Papadimitriou E: Irradiated C6 glioma cells induce angiogenesis in vivo and activate endothelial cells in vitro. Int J Cancer 110: 807-814, 2004.

12. Bartlett JB, Dredge K and Dalgleish AG: The evolution of thalidomide and its IMiD derivatives as anticancer agents. Nat Rev Cancer 4: 314-322, 2004

13. Kenyon BM, Browne F and D'Amato RJ: Effects of thalidomide and related metabolites in a mouse corneal model of neovascularization. Exp Eye Res 64: 971-978, 1997.

14. Kruse FE, Joussen AM, Rohrschneider K, Becker MD and Volcker HE: Thalidomide inhibits corneal angiogenesis induced by vascular endothelial growth factor. Graefes Arch Clin Exp Ophthalmol 236: 461-466, 1998.

15. D'Amato RJ, Loughnan MS, Flynn E and Folkman J: Thalidomide is an inhibitor of angiogenesis. Proc Natl Acad Sci USA 91: 4082-4085, 1994

16. Short SC, Traish D, Dowe A, Hines F, Gore M and Brada M: Thalidomide as an anti-angiogenic agent in relapsed gliomas. J Neurooncol 51: 41-45, 2001.

17. Fine HA, Wen PY, Maher EA, Viscosi E, Batchelor T, Lakhani N, Figg WD, Purow BW and Borkowf CB: Phase II trial of thalidomide and carmustine for patients with recurrent highgrade gliomas. J Clin Oncol 21: 2299-2304, 2003.

18. Mauceri HJ, Hanna NN, Beckett MA, Gorski DH, Staba MJ, Stellato KA, Bigelow K, Heimann R, Gately S, Dhanabal M, Soff GA, Sukhatme VP, Kufe DW and Weichselbaum RR: Combined effects of angiostatin and ionizing radiation in antitumour therapy. Nature 394: 287-291, 1998.
19. Gorski DH, Mauceri HJ, Salloum RM, Gately S, Hellman S, Beckett MA, Sukhatme VP, Soff GA, Kufe DW and Weichselbaum RR: Potentiation of the antitumor effect of ionizing radiation by brief concomitant exposures to angiostatin. Cancer Res 58: 5686-5689, 1998.

20. Newlands ES, Stevens MF, Wedge SR, Wheelhouse RT and Brock C: Temozolomide: a review of its discovery, chemical properties, pre-clinical development and clinical trials. Cancer Treat Rev 23: 35-61, 1997.

21. Stupp R, Dietrich PY, Ostermann Kraljevic S, Pica A, Maillard I, Maeder P, Meuli R, Janzer R, Pizzolato G, Miralbell R, Porchet F, Regli L, de Tribolet N, Mirimanoff RO and Leyvraz S: Promising survival for patients with newly diagnosed glioblastoma multiforme treated with concomitant radiation plus temozolomide followed by adjuvant temozolomide. J Clin Oncol 20: 1375-1382, 2002.

22. Stupp R, Mason WP, van den Bent MJ, et al: Radiotherapy plus concomitant and adjuvant temozolomide for glioblastoma. N Engl J Med 352: 987-996, 2005.

23. Nam DH, Park K, Park C, Im YH, Kim MH, Lee S, Hong SC, Shin HJ, Kim JH, Eoh W and McDonnell TJ: Intracranial inhibition of glioma cell growth by cyclooxygenase-2 inhibitor celecoxib. Oncol Rep 11: 263-268, 2004.

24. Lal S, Lacroix M, Tofilon P, Fuller GN, Sawaya R and Lang FF: An implantable guide-screw system for brain tumor studies in small animals. J Neurosurg 92: 326-333, 2000.

25. Brown CK, Khodarev NN, Yu J, Moo-Young T, Labay E, Darga TE, Posner MC, Weichselbaum RR and Mauceri HJ: Glioblastoma cells block radiation-induced programmed cell death of endothelial cells. FEBS Lett 565: 167-170, 2004.

26. Ansiaux R, Baudelet C, Jordan BF, Beghein N, Sonveaux P, De Wever J, Martinive P, Gregoire V, Feron O and Gallez B: Thalidomide radiosensitizes tumors through early changes in the tumor microenvironment. Clin Cancer Res 11: 743-750, 2005.

27. Paris F, Fuks Z, Kang A, Capodieci P, Juan G, Ehleiter D, Haimovitz-Friedman A, Cordon-Cardo C and Kolesnick R: Endothelial apoptosis as the primary lesion initiating intestinal radiation damage in mice. Science 293: 293-297, 2001

28. Son MJ, Kim JS, Kim MH, Song HS, Kim JT, Kim H, Shin T, Jeon HJ, Lee DS, Park SY, Kim YJ, Kim JH and Nam DH: Combination treatment with temozolomide and thalidomide inhibits tumor growth and angiogenesis in an orthotopic glioma model. Int J Oncol 28: 53-59, 2006.

29. Jain RK: Normalizing tumor vasculature with anti-angiogenic therapy: a new paradigm for combination therapy. Nat Med 7: 987-989, 2001.

30. Jain RK: Normalization of tumor vasculature: an emerging concept in antiangiogenic therapy. Science 307: 58-62, 2005. 